\title{
MICROEXTRAÇÃO EM FASE LÍQUIDA (LPME): FUNDAMENTOS DA TÉCNICA E APLICAÇÕES NA ANÁLISE DE FÁRMACOS EM FLUIDOS BIOLÓGICOS
}

\author{
Anderson Rodrigo Moraes de Oliveira, Igor Rafael dos Santos Magalhães, Fernando José Malagueño de Santana e \\ Pierina Sueli Bonato*
}

Faculdade de Ciências Farmacêuticas de Ribeirão Preto, Universidade de São Paulo, Av. do Café, s/n, 14040-903

Ribeirão Preto - SP, Brasil

Recebido em 1/12/06; aceito em 30/8/07; publicado na web em 26/2/08

\begin{abstract}
LIQUID-PHASE MICROEXTRACTION (LPME): FUNDAMENTALS AND APPLICATIONS TO THE ANALYSIS OF DRUGS IN BIOLOGICAL SAMPLES. The analysis of drugs and metabolites in biological fluids usually requires extraction procedures to achieve sample clean-up and analyte preconcentration. Commonly, extraction procedures are performed using liquid-liquid extraction or solidphase extraction. Nevertheless, these extraction techniques are considered to be time-consuming and require a large amount of organic solvents. On this basis, microextraction techniques have been developed. Among them, liquid-phase microextraction has been standing out. This review describes the liquid-phase microextraction technique based on hollow fibers as a novel and promising alternative in sample preparation prior to chromatographic or electrophoretic analysis. The basic concepts related to this technique and its applicability in extraction of drugs are discussed.
\end{abstract}

Keywords: liquid-phase microextraction; drug analysis; biological samples.

\section{INTRODUÇÃO}

As técnicas de separação, tais como a cromatografia líquida de alta eficiência (HPLC), a cromatografia gasosa (GC) e a eletroforese capilar (CE), são perfeitamente adequadas para a análise de amostras contendo inúmeros componentes. No entanto, a determinação de analitos presentes em matrizes complexas, tais como plasma, soro, sangue total, homogenatos de tecidos, saliva ou urina, geralmente requer processos prévios de preparação das amostras bem elaborados. ${ }^{1}$ Vários motivos justificam estes procedimentos, destacando-se a necessidade de eliminação prévia de parte dos componentes devido à complexidade das matrizes biológicas, a existência de proteínas que podem adsorver nas colunas cromatográficas ou nos capilares, além da necessidade de uma etapa de concentração das substâncias a serem analisadas, geralmente presentes em nível de traços. ${ }^{1,2}$

As técnicas mais comumente utilizadas para extração e/ou préconcentração de compostos presentes em fluidos biológicos são a extração líquido-líquido (LLE) e a extração em fase sólida (SPE). ${ }^{3}$ Contudo, as tendências atuais apontam no sentido da utilização de menores quantidades de amostras, até mesmo para análises de traços; obtenção de maior seletividade e especificidade na extração; aumento no potencial para automação ou utilização de métodos "online", reduzindo assim a operação manual; desenvolvimento de métodos menos agressivos ao meio ambiente, com menor desperdício e, o uso de quantidade mínima ou nenhuma de solventes orgânicos. ${ }^{4,5}$ Dentro deste contexto, técnicas de microextração que utilizam quantidades mínimas de solventes orgânicos e menos etapas na preparação das amostras vêm sendo desenvolvidas. Entre as principais técnicas de microextração existentes destacam-se a microextração em fase sólida (SPME), a microextração em gota suspensa (SDME) e a extração em membranas ["supported liquid membrane" (SLM), "microporous membrane liquid-liquid extraction" (MMLLE), "liquid-phase microextraction" (LPME)].

*e-mail: psbonato@fcfrp.usp.br
A SPME foi introduzida por Arthur e Pawliszyn no início da década passada. ${ }^{6}$ Nessa técnica, os processos de extração e préconcentração ocorrem através da distribuição dos analitos entre a fase extratora e a matriz. O dispositivo básico de SPME consiste de um tubo capilar de sílica fundida de $100 \mu \mathrm{m}$ de diâmetro e com a extremidade recoberta com um filme fino de um polímero ou de um sólido adsorvente. ${ }^{6-8}$ Quanto maior a afinidade do analito pela fase extratora em relação à matriz, maior será a quantidade extraída. A extração pode ser feita de maneira direta ou indireta. $\mathrm{Na}$ primeira, a fibra é mergulhada diretamente na solução da amostra que é submetida à agitação. A extração direta é empregada quando o analito não é volátil ou, no caso de analitos voláteis, quando a amostra não é tão complexa. A extração indireta ("headspace") é empregada para analitos voláteis, já que a fibra não entra em contato direto com a solução. A amostra é freqüientemente aquecida e os componentes voláteis são extraídos para a fibra pelo processo de sorção. ${ }^{8}$ No entanto, a SPME tem como principal limitação baixos valores de recuperação. ${ }^{9} \mathrm{Na}$ análise de fármacos em fluidos biológicos, principalmente em estudos farmacocinéticos, a quantidade de amostra viável para análise é, freqüentemente, muito pequena. Assim, devido a essa deficiência na recuperação, são necessários equipamentos com alta detectabilidade. Outra desvantagem relacionada ao uso da SPME para preparação de amostras submetidas à análise por HPLC é a fragilidade apresentada pelas fibras de extração perante os solventes orgânicos, sendo que somente solventes de alta polaridade (metanol, acetonitrila e misturas aquosas destes) devem ser utilizados para o processo de dessorção. ${ }^{10}$ Por fim, o número de fibras disponível comercialmente é pequeno, o que limita a seletividade da técnica. A seletividade das fibras de SPME pode ser modificada através da obtenção de polímeros pelo processo sol-gel. ${ }^{11}$ Este tipo de preparo de fibras mostrou-se muito promissor, pois os polímeros obtidos apresentaram algumas vantagens em relação à fibra de polidimetilsiloxano (PDMS) convencional (mais seletivas e estáveis termicamente e com maiores áreas superficiais). Além disso, o processo de síntese desses polímeros é mais fácil e rápido. 
Uma tendência recente na busca por novas técnicas de preparação de amostras é a miniaturização do método tradicional de LLE com o objetivo de reduzir a razão volumétrica entre a fase aquosa, chamada de doadora, e a fase orgânica, denominada de aceptora e favorecer a transferência dos analitos da fase aquosa para a fase orgânica (elevado fator de enriquecimento). Isto pode ser obtido usando tanto fases líquidas imiscíveis (microextração em gota suspensa) quanto membranas separando as fases aceptora e doadora (extração em membrana). ${ }^{11}$

$\mathrm{O}$ conceito de microextração em gota suspensa ("single drop microextraction" - SDME) foi introduzido em 1996 por Jeannot e Cantwell, a partir do desenvolvimento de uma técnica em que uma microgota de solvente orgânico imiscível em água $(\sim 50 \mu \mathrm{L})$ era acomodada na porção final de um dispositivo de politetrafluoretileno (PTFE), sendo então imerso na solução contendo a amostra. ${ }^{12}$ Após a extração sob agitação, o dispositivo era retirado da amostra e a fase orgânica injetada no cromatógrafo a gás. Posteriormente, a técnica foi modificada, passando-se a utilizar uma microsseringa como suporte, onde a fase orgânica era suspensa na ponta da agulha (Figura 1A). Tal sistema também possibilita o uso de três fases; neste caso, microlitros de fase aquosa estão contidos no interior da seringa (anterior à gota de solvente orgânico). ${ }^{13}$

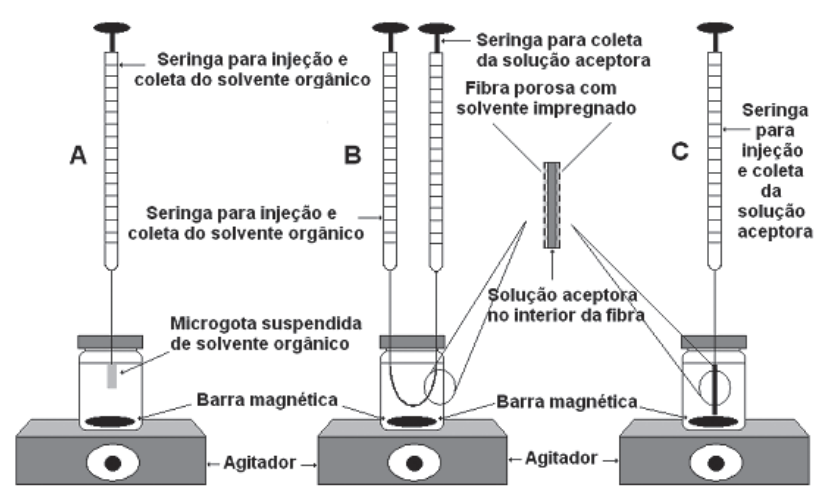

Figura 1. Representação esquemática da microextração em gota suspensa (SDME) (A) e da microextração em fase líquida (LPME) empregando configuração em " $U$ " (B) e do tipo "haste" ("rod-like") (C)

Nesta técnica, os analitos de elevado coeficiente de partição podem atingir altas concentrações, uma vez que são transferidos por difusão de um expressivo volume de amostra (1-5 mL) para um diminuto microextrato $(5-50 \mu \mathrm{L})$. No entanto, a microextração em gota suspensa não é tão robusta, ocorrendo perda de solvente durante a extração, especialmente quando é aplicada agitação para acelerar o processo de extração, além da necessidade de pré-tratamento de amostras bastante viscosas ou contendo material particulado (possível colisão com a microgota). ${ }^{14}$

A extração em membranas, por sua vez, é mais robusta e apresenta como características mais importantes: o alto grau de seletividade, que possibilita a obtenção de extratos livres de interferentes, a capacidade de concentrar os analitos, permitindo a análise de substâncias em níveis de traços em matrizes complexas e a significativa redução no consumo de solventes orgânicos. ${ }^{15,16}$ A técnica denominada extração com fase líquida suportada em membranas ("supported liquid membrane" - SLM) pode ser considerada uma extração líquido-líquido em duas etapas e baseia-se em um sistema de três fases, no qual a fase orgânica se encontra entre duas fases aquosas. A fase orgânica é imobilizada em uma membrana hidrofóbica porosa, a qual forma uma barreira entre as fases aquosas. Solventes tipicamente usados incluem hidrocarbonetos de ca- deia longa, como $n$-undecano, ou ainda mais polares como éter diexílico e tri-octilfosfato..$^{15} \mathrm{Na}$ SLM, os analitos de interesse presentes na fase doadora são mantidos na sua forma não-ionizada através de ajustes no $\mathrm{pH}$ e, dessa forma, são transferidos através da membrana impregnada de solvente orgânico para a fase aceptora também aquosa que, por sua vez, apresenta um $\mathrm{pH}$ contrário àquele da fase doadora, o que favorece a formação de moléculas ionizadas do analito, impedindo seu regresso para a fase orgânica. Após a extração, a fase aceptora é transferida para o instrumento analítico, por exemplo, HPLC em fase reversa ou CE e assim analisado. Compostos neutros estarão nas três fases, porém não serão concentrados e a taxa de extração de macromoléculas será baixa, devido ao baixo coeficiente de difusão promovido pelo uso de membranas com poros na ordem de $0,2 \mu \mathrm{m}$ de diâmetro. ${ }^{15,17}$

Outra técnica de extração por membranas, denominada extração líquido-líquido em membrana microporosa ("microporous membrane liquid-liquid extraction" - MMLLE) possui o mesmo princípio da LLE, porém é realizada sob fluxo constante, o qual facilita a automação e interfaciamento com os instrumentos analíticos. Nesta técnica, a fase aceptora é um solvente orgânico, o qual também preenche os poros da membrana, formando então um sistema de duas fases. Assim como na LLE convencional, a eficiência da extração é limitada pelo coeficiente de partição $(K)$ do analito. A MMLLE é mais adequada para compostos altamente hidrofóbicos, os quais encontrariam dificuldades de serem extraídos em uma fase aceptora aquosa. ${ }^{15,17,18}$

Desvantagens em potencial dessas técnicas (SLM e MMLLE) incluem problemas de estabilidade da membrana e possibilidade de ocorrer "carry-over" (efeitos de memória), por serem utilizadas em várias extrações; por outro lado, podem ser facilmente utilizadas "on-line" com os equipamentos de análise. ${ }^{17,19}$

\section{MICROEXTRAÇÃO EM FASE LÍQUIDA COM FIBRAS OCAS}

Pedersen-Bjergaard e Rasmussen idealizaram uma nova metodologia que combina o conceito de extrações com membranas (SLM e MMLLE) com o uso reduzido da razão solvente orgânico/fase aquosa, visto na $\mathrm{SDME}^{20}$. Esta técnica (microextração em fase líquida com fibras ocas, "hollow fiber liquid-phase microextraction" - HFLPME, ou simplesmente, LPME) pode ser considerada uma evolução dos métodos de microextração com solventes. Os poros de uma membrana capilar porosa e hidrofóbica (fibra oca) (Figura 2) são impregnados com o solvente orgânico de extração e o seu lúmen é preenchido com microlitros de uma fase aceptora. Com isso, a fase aceptora não entra em contato direto com a matriz aquosa (fase doadora), permitindo aplicar agitação constante durante a extração. Além disso, o baixo custo de cada unidade de extração possibilita o seu uso uma única vez, evitando problemas de "carry-over", efeito normalmente observado em outras técnicas de extração com membranas. ${ }^{20,21}$

Os fundamentos da LPME são similares aos empregados em SLM e MMLLE, porém as técnicas diferem em termos de instrumentação e operação. As técnicas citadas anteriormente funcionam através de procedimentos operacionais com sistemas de fluxo constante, pelo emprego de bombas peristálticas que renovam continuamente os compartimentos próximos à membrana. Por outro lado, nenhum aparato específico é necessário para implantação da LPME. Há duas configurações principais em que a LPME é empregada: configuração em "U" (Figura 1B), que utiliza duas microsseringas conectadas à fibra (mais empregada) e, configuração tipo "haste" ("rod-like"), onde somente uma microsseringa é utilizada para injetar e coletar a fase aceptora ${ }^{22}$ (Figura 1C).

A LPME pode ser utilizada em dois modos: duas ou três fases, de acordo com as características do analito em questão (Figura 2A e 
A

Solvente orgânico impregnado nos poros

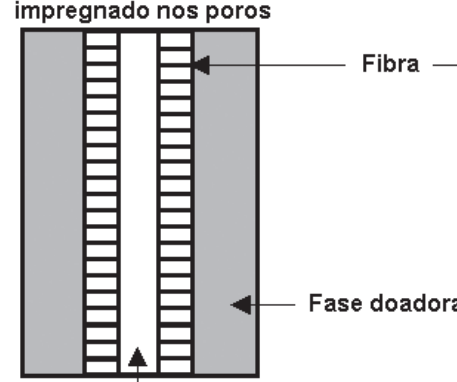

Fase aceptora orgânica
B

Solvente orgânico impregnado nos poros $\square$

Figura 2. Modos de extração utilizados na microextração em fase líquida (LPME): duas fases (A) e três fases $(B)$

B). No sistema de duas fases (Figura 2A), o analito é extraído da amostra aquosa (fase doadora) através de um solvente orgânico imiscível em água imobilizado nos poros da membrana passando para o mesmo solvente orgânico (fase aceptora), presente no lúmen da fibra. Para obtenção de resultados favoráveis neste modo de extração, o analito deverá ser moderada ou altamente hidrofóbico, podendo conter grupos ionizáveis ácidos ou básicos. ${ }^{21}$

No modo de três fases (Figura 2B), o analito é extraído de uma amostra aquosa (fase doadora) através de um solvente orgânico imiscível em água imobilizado nos poros da membrana, passando para uma solução aquosa (fase aceptora) presente no lúmen da fibra. A fase orgânica atua como uma barreira entre as fases aceptora e doadora (ambas aquosas), impedindo o contato entre as duas fases. ${ }^{21}$

Para analitos altamente hidrofílicos, que encontrariam dificuldades em serem extraídos na membrana orgânica apenas pelo processo de difusão, uma outra possibilidade seria a adição de reagente iônico relativamente hidrofóbico à fase doadora ou orgânica, permitindo a formação de par iônico com o analito de interesse, ocorrendo então a extração. ${ }^{23-25}$ Recentemente, Yazdi e Es'haghi relataram o emprego de tensoativos não-iônicos (ex.: Triton X-100 ${ }^{\circledR}$ ) adicionados na fase doadora para determinação de drogas de abuso hidrofílicas no cabelo. ${ }^{26}$

\section{Recuperação e fator de enriquecimento}

Em sistemas de duas fases, a transferência dos analitos da fase aquosa para a fase orgânica pode ser assim descrita: ${ }^{27}$

A (fase doadora) $\leftrightarrows \mathrm{A}$ (fase aceptora)

onde A representa o analito de interesse. Nesse caso, o coeficiente de partição do analito entre a fase orgânica e doadora $\mathrm{K}_{\mathrm{org} / \mathrm{d}}$ é dado por:

$\mathrm{K}_{\text {org } / \mathrm{d}}=\frac{\mathrm{C}_{\mathrm{eq}, \mathrm{org}}}{\mathrm{C}_{\mathrm{eq}, \mathrm{d}}}$

onde $\mathrm{C}_{\text {eq,org }}$ é a concentração de $\mathrm{A}$ na fase aceptora no equilíbrio e $\mathrm{C}_{\text {eq,d }}$ é a concentração de A na fase doadora no equilíbrio.

A recuperação $(\mathrm{R})$ pode ser definida como a quantidade total de analito, em porcentagem, que é transferida para a fase aceptora ao final da extração; além do coeficiente de partição, a razão entre o volume da fase doadora $\left(\mathrm{V}_{\mathrm{d}}\right)$ e a fase aceptora $\left(\mathrm{V}_{\text {org }}\right)$ também governa a recuperação do analito, de acordo com a expressão:

$R=\frac{K_{\text {org } / d} V_{\text {org }}}{K_{\text {org } / d} V_{\text {org }}+V_{\text {org }}} \times 100$

Sendo assim, conclui-se que a extração em duas fases é favorecida para analitos hidrofóbicos, ou seja, com elevados coeficientes de partição (500-1000 ou maiores). ${ }^{27}$ Adicionalmente, a recuperação pode ser favorecida aumentando-se a razão entre as fases aceptora e doadora, utilizando-se fibras mais longas ou com maior diâmetro interno.

Para LPME de duas fases, a recuperação real é menor que aquela calculada pela equação acima pois a fração de solvente orgânico que se encontra imobilizado nos poros da fibra oca microporosa não está disponível para análises posteriores; somente a fração presente no lúmen pode ser coletada pela microsseringa.

Outro parâmetro comumente usado para demonstrar a eficiência dos processos de LPME é o fator de enriquecimento, que informa o grau de concentração do analito que ocorreu durante a extração.

$\mathrm{O}$ enriquecimento (E) de um analito pode ser calculado pela fórmula:

$E=\frac{V_{d} R}{100 V_{\text {org }}}$

Como os valores de $\mathrm{V}_{\mathrm{d}} / \mathrm{V}_{\text {org }}$ são normalmente elevados, altos fatores de enriquecimento são obtidos. ${ }^{22}$

No sistema de três fases, o processo de transferência dos analitos é definido como:

A (fase doadora) $\leftrightarrows$ A (fase orgânica) $\leftrightarrows$ A (fase aceptora)

Nesse sistema, deve-se considerar o coeficiente de partição entre a fase orgânica e a fase doadora, bem como entre a fase aceptora e a fase orgânica:

$$
\begin{gathered}
\mathrm{K}_{\text {org /d }}=\frac{\mathrm{C}_{\mathrm{eq}, \mathrm{rrg}}}{\mathrm{C}_{\mathrm{eq}, \mathrm{d}}} \\
\mathrm{K}_{\mathrm{a} / \text { org }}=\frac{\mathrm{C}_{\mathrm{eq}, \mathrm{a}}}{\mathrm{C}_{\mathrm{eq}, \mathrm{org}}}
\end{gathered}
$$

onde $\mathrm{C}_{\text {eq.a }}$ é a concentração do analito na fase aceptora quando o equilíbrio é atingido. O coeficiente de partição entre a fase aceptora e a fase doadora $\mathrm{K}_{\mathrm{a} / \mathrm{d}}$ pode ser escrito como:

$$
K_{a / d}=K_{o r g / d} K_{a / o r g}
$$

A recuperação $(\mathrm{R})$ pode ser expressa como:

$$
R=\frac{100 K_{a / d} V_{a}}{K_{a / d} V_{a}+K_{o r g / d} V_{o r g}+V_{d}}
$$

Nos casos de LPME de três fases, o volume total de fase aceptora está disponível para a análise e a recuperação é, portanto, diretamente calculada pela equação acima. No sistema de três fases, o solvente orgânico deve ser selecionado para ter altos $\mathrm{K}_{\text {org/d }}$ e $\mathrm{K}_{\mathrm{a} / \mathrm{org}}$; esse último valor é importante para evitar que o analito fique retido na fase orgânica, diminuindo assim sua recuperação.

$\mathrm{O}$ enriquecimento (E) pode ser calculado pela fórmula:

$E=\frac{V_{d} R}{100 V_{a}}$

A Figura 3 correlaciona a recuperação com o coeficiente de partição $\mathrm{K}_{\mathrm{org} / \mathrm{d}}$ em sistemas de 2 e 3 fases. $^{27}$ 


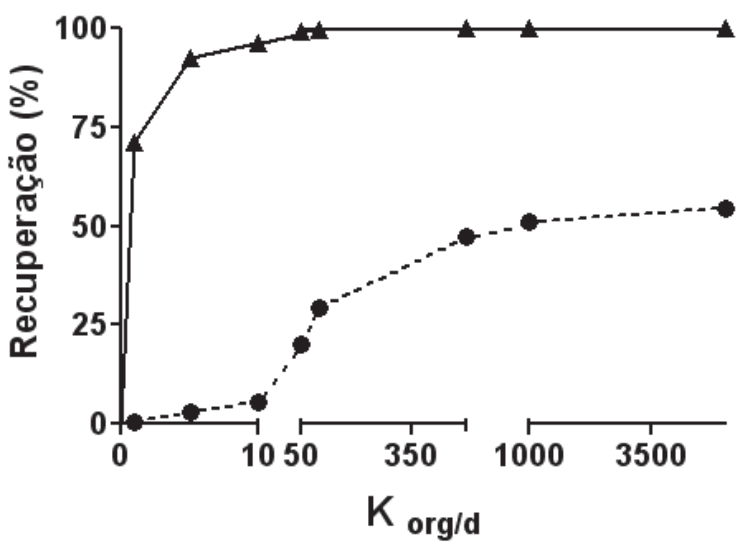

Figura 3. Correlação entre a recuperação e o coeficiente de partição $\left(K_{\mathrm{org} / d}\right)$ em sistemas de $2(\mathbf{O})$ e 3 fases $(\mathbf{\Delta})$

\section{Parâmetros que afetam a extração em LPME}

Além das características inerentes do analito (coeficientes de ionização e partição) e da razão das fases aceptora e doadora, alguns outros parâmetros devem ser considerados durante o desenvolvimento do método, tais como ajuste do $\mathrm{pH}$ da amostra, força iônica, membrana hidrofóbica, tipo de solvente orgânico, tempo e temperatura de extração, composição da solução aceptora e agitação do sistema. ${ }^{21} \mathrm{~A}$ influência desses parâmetros será discutida a seguir.

\section{Membrana hidrofóbica}

As membranas capilares empregadas em LPME devem ser hidrofóbicas e compatíveis com os solventes orgânicos utilizados. Tais requisitos são cumpridos pelas membranas baseadas em polipropileno, disponíveis comercialmente (Membrana, Wuppertal, Alemanha). ${ }^{28}$ Destas, a mais empregada apresenta diâmetro interno de $600 \mu \mathrm{m}$, compatível com os microlitros de fase aceptora; parede com espessura de $200 \mu \mathrm{m}$, oferecendo excelente estabilidade mecânica durante a extração e poros com tamanho de $0,2 \mu \mathrm{m}$, o que fornece eficaz microfiltração, impedindo a passagem de macromoléculas (ex.: proteínas). ${ }^{29}$

\section{Solvente orgânico}

Uma etapa fundamental na otimização de métodos na LPME é a seleção do solvente orgânico. Algumas propriedades são necessárias para um solvente orgânico ser empregado em LPME, dentre elas: baixa solubilidade ou insolubilidade em água, prevenindo a dissolução da fase orgânica na aquosa (doadora); baixa volatilidade, evitando a perda de fase orgânica durante a extração; compatibilidade com a membrana capilar utilizada (no caso, polipropileno); fácil impregnação nos poros da mesma e solubilidade adequada para o analito de interesse, como discutido anteriormente. ${ }^{21,22}$ Vários solventes orgânicos têm sido utilizados, como éter diexílico, $n$ octanol, acetato de hexila, acetato de butila, acetato de dodecila, éter dibutílico e isooctanol, ou misturas desses solventes. ${ }^{22}$ Dentre todos esses solventes, o éter diexílico tem mostrado excelentes resultados para inúmeras aplicações (Tabela 1). De maneira interessante, Pedersen-Bjergaard e Rasmussen avaliaram o emprego de óleos vegetais como fases orgânicas em LPME. ${ }^{30}$ Todos os óleos essenciais testados foram compatíveis com urina, porém apenas o óleo de erva-doce forneceu bons resultados na análise de plasma. Com isso, há uma nova alternativa para as fases orgânicas, eliminando-se o uso de solventes prejudiciais de uso comum.

\section{Tempo de extração}

A eficiência da extração na LPME depende da transferência de massa do analito da fase aquosa para a orgânica e desta para a fase aceptora (no caso de sistemas em três fases). É um processo de equilíbrio e, portanto, a recuperação do analito aumenta com o tempo de extração até atingir uma situação de platô. Neste ponto, o equilíbrio é atingido e a distribuição do analito entre as fases permanece constante. ${ }^{22,31} \mathrm{O}$ tempo necessário para atingir o equilíbrio pode ser reduzido aumentando a área superficial de contato da fibra com a amostra. ${ }^{32}$ Muitas vezes, o tempo necessário para que o equilíbrio seja atingido é excessivamente longo; nesses casos, a extração é feita em condições de não equilíbrio, controlando-se precisamente o tempo de extração. ${ }^{22}$

\section{Temperatura}

Com o aumento da temperatura, os coeficientes de difusão aumentam em resposta à diminuição da viscosidade. Logo, o tempo requerido para alcançar o equilíbrio é diminuído. Por outro lado, os coeficientes de partição para a fase aceptora diminuem, reduzindo a quantidade de fármaco extraído no estado de equilíbrio. Portanto, a velocidade das extrações pode ser melhorada a custa da perda da detectabilidade do método. Contudo, a LPME normalmente é executada à temperatura ambiente, evitando-se possíveis problemas de formação de bolhas e evaporação do solvente durante a extração, já que a quantidade de solvente utilizada é muito pequena (aproximadamente $20 \mu \mathrm{L}){ }^{33}$

\section{Agitação}

Normalmente, a agitação da amostra é aplicada para acelerar a cinética de extração, facilitando a difusão dos analitos através da interface fase doadora - solvente orgânico. Logo, diminui-se o tempo necessário para o sistema atingir o equilíbrio. ${ }^{34}$

$\mathrm{Na}$ SDME, o uso de velocidades de agitação elevadas (acima de $600 \mathrm{rpm}$ ) provoca a perda da fase aceptora presente na ponta da agulha, prejudicando a quantificação dos analitos. Por outro lado, na LPME, a fase aceptora está contida no interior da fibra cilíndrica, possibilitando o emprego de velocidades mais elevadas de agitação. ${ }^{35}$ Nesse sentido, pode-se empregar agitação magnética ou banho ultra-sônico para a agitação da amostra.

Embora utilizada em diversos trabalhos, a agitação induzida por uma barra magnética pode provocar contaminação cruzada das amostras e a formação de bolhas de ar que tendem a aderir na superfície da fibra cilíndrica, acelerando a evaporação do solvente e introduzindo imprecisão nas medições. ${ }^{35,36}$

\section{pH da fase doadora}

$\mathrm{O}$ pH da amostra é crucial para a eficiente extração de compostos ácidos e básicos. $\mathrm{O}$ ajuste do $\mathrm{pH}$ resulta em uma maior razão de distribuição e garante elevados fatores de enriquecimentos e valores de recuperação do analito de interesse. ${ }^{36}$ Ajustes no $\mathrm{pH}$ podem aumentar a eficiência da extração, uma vez que tanto o equilíbrio de dissociação quanto a solubilidade de ácidos e bases são diretamente afetados pelo $\mathrm{pH}$ da amostra. ${ }^{37}$

\section{Aditivos na fase doadora}

\section{$\underline{\text { Sais }}$}

O efeito da adição de sais à matriz é complexo. Pode aumentar a eficiência da extração, particularmente para analitos mais polares, devido ao efeito denominado "salting-out". ${ }^{21}$ Neste processo, as moléculas de água da fase doadora passam a hidratar também os íons adicionados ocorrendo, então, a redução de moléculas do analito dissolvidos na solução aquosa por mecanismos de compe- 
Tabela 1. Aplicações da LPME na análise de fármacos em fluidos biológicos

\begin{tabular}{|c|c|c|c|c|c|c|}
\hline Fármaco & Amostra & Solvente & Fase aceptora & Tipo de extração & Sistema analítico ${ }^{\mathrm{a}}$ & Ref. \\
\hline Aminoálcoois & Urina & n-octanol & $\mathrm{HCl} 0,1 \mathrm{~mol} \mathrm{~L}^{-1}$ & Três fases & CE-UV & 68 \\
\hline $\begin{array}{l}\text { Anfetamina e } \\
\text { análogos }\end{array}$ & $\begin{array}{c}\text { Sangue total e } \\
\text { urina }\end{array}$ & Éter diexílico & $\mathrm{HCl} 0,01 \mathrm{~mol} \mathrm{~L}^{-1}$ & Três fases & MS & 57 \\
\hline Antidepressivos & $\begin{array}{c}\text { Plasma e } \\
\text { sangue total }\end{array}$ & $\begin{array}{l}\text { Acetato de } \\
\text { dodecila }\end{array}$ & $\mathrm{HCOOH} 0,2 \mathrm{~mol} \mathrm{~L}^{-1}$ & Três fases & HPLC-MS & 58 \\
\hline Antidepressivos & Leite materno & $\begin{array}{l}\text { Polifenilme- } \\
\text { tilsiloxano }\end{array}$ & $\mathrm{HCl} 0,01 \mathrm{~mol} \mathrm{~L}^{-1}$ & Três fases & CE-UV & 65 \\
\hline Bifenilas policloradas & Plasma & Tolueno & Tolueno & Duas fases & GC-MS & 52 \\
\hline $\begin{array}{l}\text { Citalopram e } \\
n \text {-desmetilcitalopram } \\
\text { (enantiômeros) }\end{array}$ & Plasma & $\begin{array}{l}\text { Acetato de } \\
\text { dodecila }\end{array}$ & $\begin{array}{l}\text { Tampão fosfato } \\
0,02 \mathrm{~mol} \mathrm{~L}^{-1} \\
(\mathrm{pH} \mathrm{2,75)}\end{array}$ & Três fases & CE-UV & 53 \\
\hline $\begin{array}{l}\text { Citalopram e } \\
n \text {-desmetilcitalopram }\end{array}$ & Plasma & Éter diexílico & $\begin{array}{l}\text { Tampão fosfato } \\
0,02 \mathrm{~mol} \mathrm{~L}^{-1} \\
(\mathrm{pH} \mathrm{2,75)}\end{array}$ & Três fases & CE-UV & 69 \\
\hline $\begin{array}{l}\text { Citalopram e } \\
n \text {-desmetilcitalopram }\end{array}$ & Plasma & $n$-octanol & $\begin{array}{l}\text { Tampão fosfato } \\
0,01 \mathrm{~mol} \mathrm{~L}^{-1} \\
(\mathrm{pH} \mathrm{3})\end{array}$ & Três fases & HPLC-F & 70 \\
\hline Cocaína e metabólitos & Saliva e urina & Clorofórmio & Clorofórmio & Duas fases & GC-PDHID & 62,66 \\
\hline $\begin{array}{l}\text { Diazepam e } \\
n \text {-desmetildiazepam }\end{array}$ & Plasma e urina & $\begin{array}{l}\text { Acetato de butila: } \\
n \text {-octanol (1:1) ou } \\
\text { éter diexílico: } \\
n \text {-octanol (1:3) }\end{array}$ & $\begin{array}{l}\text { Acetato de butila: } \\
\text { n-octanol (1:1) ou } \\
\text { éter diexílico: } \\
n \text {-octanol }(1: 3)\end{array}$ & Duas fases & GC-NPD & 50 \\
\hline Diazepam e prazepam & Plasma & n-octanol & n-octanol & Duas fases & GC-NPD & 70 \\
\hline Esteróides anabolizantes & Urina & n-octanol & $\mathrm{NH}_{3} 0,25 \mathrm{~mol} \mathrm{~L}^{-1}$ & Três fases & HPLC-MS & 44 \\
\hline Esteróides anabolizantes & Urina & $n$-octanol & n-octanol & Duas fases & HPLC-MS e GC-MS & 71 \\
\hline $\begin{array}{l}\text { Fenilpropanolamina } \\
\text { e practolol }\end{array}$ & Plasma & $n$-octanol & $\mathrm{HCl} 0,05 \mathrm{~mol} \mathrm{~L}^{-1}$ & Três fases & CE-UV & 25 \\
\hline $\begin{array}{l}\text { Haloperidol, metadona } \\
\text { e prometazina }\end{array}$ & Plasma e urina & Éter diexílico & $\mathrm{HCl} 0,01 \mathrm{~mol} \mathrm{~L}^{-1}$ & Três fases & GC-FID e CE-UV & 27 \\
\hline $\begin{array}{l}\text { Hidroxicloroquina e } \\
\text { metabólito (enantiômeros) }\end{array}$ & Urina & $n$-octanol & $\mathrm{HCl} 0,1 \mathrm{~mol} \mathrm{~L}^{-1}$ & Três fases & CE-UV & 61 \\
\hline $\begin{array}{l}\text { Ibuprofeno, cetoprofeno } \\
\text { e naproxeno }\end{array}$ & Urina & Éter diexílico & $\mathrm{NaOH} 0,01-0,1 \mathrm{~mol} \mathrm{~L}^{-1}$ & Três fases & CE-UV & 36 \\
\hline Mefloquina (enantiômeros) & Plasma & Éter diexílico & $\mathrm{HClO}_{4} 0,01 \mathrm{~mol} \mathrm{~L}^{-1}$ & Três fases & HPLC-UV & 55 \\
\hline Metanfetamina & Plasma e urina & n-octanol & $\mathrm{HCl}^{4} 0,1 \mathrm{~mol} \mathrm{~L}^{-1}$ & Três fases & CE-UV & 20 \\
\hline $\begin{array}{l}\text { Metanfetamina } \\
\text { e citalopram }\end{array}$ & $\begin{array}{l}\text { Plasma, urina e } \\
\text { sangue total }\end{array}$ & Éter diexílico & $\mathrm{HCl} 0,1 \mathrm{~mol} \mathrm{~L}^{-1}$ & Três fases & HPLC-UV & 33 \\
\hline Mianserin (enantiômeros) & Plasma & Éter diexílico & $\mathrm{HCl} 0,01 \mathrm{~mol} \mathrm{~L}^{-1}$ & Três fases & CE-UV & 29 \\
\hline Mirtazapina (enantiômeros) & Plasma & Tolueno & Tolueno & Duas fases & HPLC-UV & 54 \\
\hline $\begin{array}{l}\text { Noradrenalina, atenolol } \\
\text { e pindolol }\end{array}$ & Urina & $n$-octanol & $\mathrm{HCl} 0,1 \mathrm{~mol} \mathrm{~L}^{-1}$ & Três fases & CE-UV & 68 \\
\hline Opióides & Cabelo & n-octanol & $\mathrm{HCl}, \mathrm{pH} 2$ & Três fases & HPLC-UV & 26 \\
\hline Salbutamol e terbutalina & Urina & Éter diexílico & $\mathrm{NaBr} 1 \mathrm{~mol} \mathrm{~L}^{-1}$ & Três fases & HPLC-MS & 72 \\
\hline THC-COOH & Urina & n-octano & n-octano & Duas fases & GC-MS & 41 \\
\hline
\end{tabular}

${ }^{\mathrm{a} C E}-\mathrm{UV}$ : eletroforese capilar-especfototrometria por absorção no UV-Vis; MS: espectrometria de massas; HPLC-MS: cromatografia líquida de alta eficiência-espectrometria de massas; GC-MS: cromatografia gasosa-espectrometria de massas; HPLC-F: cromatografia líquida de alta eficiência-espectrofotometria de fluorescência; GC-PHID: cromatografia gasosa-detector de ionização por descarga pulsada de hélio; GC-NPD: cromatografia gasosa-detector de nitrogênio e fósforo; GC-FID: cromatografia gasosa-detector por ionização em chama; HPLCUV: cromatografia líquida de alta eficiência-espectrofotometria por absorção no UV-Vis; THC-COOH: $\Delta^{9}$-carboxi-tetraidrocanabinol.

tição. ${ }^{38}$ Por outro lado, a interação das moléculas do analito com os íons adicionados, pode reduzir a difusão do analito para a fase extratora; ${ }^{35}$ além disso, o aumento da viscosidade da matriz pode reduzir a mobilidade dos analitos e prejudicar também a extração. ${ }^{39}$ Entre os sais empregados como aditivos na fase doadora, o cloreto de sódio é o mais comum.

\section{Modificadores orgânicos}

Muitos fármacos estão ligados às proteínas das matrizes biológicas de forma bastante intensa e essas interações podem ser responsáveis por baixos valores de recuperação. ${ }^{40}$ As ligações fármaco-proteínas ocorrem devido a interações iônicas, hidrofóbicas ou mesmo polares.

As interações hidrofóbicas podem ser suprimidas pela adição de alguns solventes orgânicos à matriz contendo o fármaco de interesse. Solventes orgânicos (ex.: metanol) têm a habilidade de quebrar interações hidrofóbicas e polares. ${ }^{22}$ Por outro lado, o metanol pode afetar a distribuição do analito entre a fase aquosa e o solvente orgânico, reduzindo a eficiência da extração. 


\section{Reagentes para formação de pares iônicos}

Há duas formas de emprego desta técnica na extração por LPME: adição de um reagente aniônico $\left(\mathrm{Q}^{-}\right)$ou adição de um reagente catiônico $\left(\mathrm{Q}^{+}\right)$à amostra. ${ }^{25}$ Nesses casos, o $\mathrm{pH}$ terá um papel crucial na extração. Dentre os reagentes catiônicos, as aminas quaternárias contendo cadeias alquila (conferem maior hidrofobicidade) são bastante utilizadas para extração de compostos carboxilados. ${ }^{41}$ Para análise de compostos de caráter básico, ácidos octanóico e decanóico ou sais destes vêm sendo utilizados com sucesso. ${ }^{23}$ Mais recentemente, Reubsaet et al. $^{42}$ relataram a extração de aminas biogênicas (angiotensina e neurotensina) em plasma empregando ácido heptanosulfônico como reagente iônico. Uma característica marcante deste tipo de extração é o uso de álcoois lipofílicos de cadeia longa (ex.: n-octanol) como fase orgânica devido ao caráter polar residual dos pares iônicos formados, requerendo solventes de média polaridade para eficaz solvatação.

\section{Fase aceptora no sistema de três fases}

Na LPME de três fases direcionada para extração de fármacos de caráter básico, o $\mathrm{pH}$ da fase aceptora deverá estar na faixa ácida (diferentemente da fase doadora), promovendo eficaz protonação dos analitos, evitando-se assim a retenção na fase orgânica e favorecendo a partição na fase aceptora aquosa. ${ }^{39,43}$ Nesse modo trifásico empregado para fármacos básicos, diversas soluções ácidas vêm sendo utilizadas, como os ácidos clorídrico, acético, fórmico, nítrico, sulfúrico e trifluoracético. ${ }^{37}$ Nesses sistemas, os ácidos fortes vêm se destacando, resultando em melhores valores de recuperação quando comparados com soluções aceptoras contendo ácidos fracos. Tal melhora é atribuída principalmente ao menor $\mathrm{pH}$ e eficaz protonação dos analitos obtidos no emprego dessas soluções. Para extração de compostos ácidos, soluções de hidróxido de sódio 0,01-0,1 mol L-1 vêm sendo empregadas como fases aceptoras. ${ }^{36}$ Além disso, pode-se utilizar fases aceptoras constituídas por soluções-tampão ajustadas no $\mathrm{pH}$ de interesse. Em alguns estudos empregando LPME em três fases, a adição de modificadores orgânicos (metanol ou etanol) melhorou a solubilidade do analito na solução aquosa, promovendo então aumento na extração. ${ }^{37,44}$ No entanto, deve-se atentar para a quantidade de solvente utilizada, pois em maiores concentrações foi verificada evaporação e conseqüente prejuízo na extração. ${ }^{44}$

\section{Limitações da LPME}

Como qualquer outra técnica de extração, a LPME também apresenta algumas limitações. Analitos altamente polares e hidrofílicos são difíceis de serem extraídos, sendo freqüentemente necessária a adição de par-iônico na amostra. Além disso, somente solventes com alto ponto de ebulição e insolúveis em água devem ser utilizados, limitando um pouco a seletividade do método. Uma possível desvantagem atribuída à LPME é a perda de precisão ocasionada pela operação manual do procedimento incluindo a preparação, impregnação e acomodação da fibra no sistema, além do fato de se trabalhar com um pequeno volume de fase aceptora ${ }^{45}$ Logo, é fundamental o emprego da padronização interna na quantificação das amostras. A LPME ainda é normalmente feita de forma manual, embora existam algumas tentativas de utilização "on-line" em equipamentos de HPLC e CE. ${ }^{46,47}$

\section{Aplicações da LPME na extração de fármacos de matrizes biológicas}

Diversos trabalhos vêm sendo publicados utilizando a LPME como técnica de extração em amostras biológicas. A Tabela 1 resume as mais importantes aplicações da técnica efetuadas em plasma, sangue total, urina, leite materno, saliva e cabelo.

\section{Plasma}

Geralmente, o plasma é a amostra biológica preferida para fins de monitorização terapêutica, pois a concentração do analito nesta matriz (ou no sangue total) informa a exposição direta dos tecidos ao fármaco em questão. ${ }^{48}$ Em relação à composição, o plasma humano contém aproximadamente $7-8 \%$ de proteínas, sendo que a albumina corresponde a $55 \%$ do total, além de $\alpha_{1}$-glicoproteína ácida, lipoproteínas e imunoglobulinas, entre outras. ${ }^{49}$ Além da fração protéica, outras substâncias também são encontradas: eletrólitos, lipídeos, hormônios e metabólitos, cujas concentrações estão sujeitas a variações fisiológicas ou patológicas. ${ }^{38}$ Conforme observado, a riqueza de substâncias presentes neste fluido biológico torna a análise de fármacos difícil devido aos interferentes endógenos ou exógenos, bem como devido à ligação de fármacos às proteínas plasmáticas.

No final da década passada, Pedersen-Bjergaard e Rasmussen relataram o primeiro emprego da LPME na análise de amostras de plasma tendo metanfetamina como analito de estudo. ${ }^{20}$ Os autores obtiveram um fator de enriquecimento de 75 com limite de quantificação de $20 \mathrm{ng} \mathrm{mL}^{-1}$ usando um sistema de três fases (noctanol:ácido clorídrico) e análise por CE-UV. Mais tarde, Ugland et $a l^{50}$ demonstraram o emprego da técnica para determinação de diazepam e seu principal metabólito ( $n$-desmetildiazepam) em plasma. Neste sistema de duas fases tendo éter diexílico:n-octanol (1:3) como fase orgânica, $1 \mu \mathrm{L}$ do extrato era diretamente injetado no GC-NPD ("gas chromatography nitrogen phosphorus detection"), dispensando a necessidade de evaporação e reconstituição das amostras antes da injeção.

A ligação dos fármacos às proteínas constitui uma das dificuldades encontradas na determinação destes analitos em amostras de plasma. Neste sentido, Ho et al. ${ }^{51}$ estudaram o efeito de diferentes estratégias para supressão destas interações e observaram que, dependendo das características do analito, a adição de metanol em diferentes proporções pode ser útil. Outros trabalhos envolvendo a análise de fármacos no plasma são descritos na literatura (Tabela 1). Além de fármacos, a técnica também pode ser utilizada para determinação de poluentes ambientais em plasma, conforme descrito por Basheer et al. ${ }^{52}$ os quais desenvolveram um método para determinação de bifenilas policloradas, compostos bastante lipofílicos, empregando apenas $3 \mu \mathrm{L}$ de tolueno para extração em um sistema de duas fases.

Outro interesse crescente é a análise enantiosseletiva de fármacos tendo a LPME como técnica de preparo de amostras. ${ }^{29}$ Andersen et al. desenvolveram um método para quantificação dos enantiômeros do mianserin em plasma utilizando a técnica em três fases (éter diexílico:ácido clorídrico $0,01 \mathrm{M}$ ) associada à $\mathrm{CE}$ ( $\beta$-ciclodextrina hidroxipropilada como seletor quiral). ${ }^{29}$ Ótimos valores de recuperação foram obtidos (aproximadamente 85\%) e a fase aceptora era perfeitamente compatível com o sistema analítico, propiciando a injeção direta do extrato. O mesmo grupo aplicou um sistema de três fases (acetato de dodecila:tampão fosfato $20 \mathrm{mM}, \mathrm{pH} 2,75$ ) juntamente à $\mathrm{CE}$ ( $\beta$-ciclodextrina sulfatada como seletor quiral) para análise dos enantiômeros do citalopram e $n$-desmetilcitalopram em plasma, com limite de quantificação de $11,2 \mathrm{ng} \mathrm{mL}^{-1} .^{53}$

Em nosso grupo, De Santana et al.$^{54}$ desenvolveram um método para determinação dos enantiômeros da mirtazapina em plasma utilizando um sistema de duas fases (solvente orgânico - tolueno) associado à HPLC com fase estacionária quiral, com limite de quantificação de $6 \mathrm{ng} \mathrm{mL}^{-1}$. Mais tarde, Magalhães e Bonato ${ }^{55}$ obtiveram valores de recuperação em torno de $35 \%$ na análise dos enantiômeros da mefloquina em plasma humano e de ratos, utilizando um sistema de três fases (éter diexílico:ácido perclórico $0,01 \mathrm{~mol} \mathrm{~L}^{-1}$ ) com excelente seletividade frente aos interferentes endógenos. 
De maneira geral, métodos desenvolvidos para análise de plasma podem ser aplicados para amostras de soro sem nenhuma modificação prévia. ${ }^{56}$

\section{Sangue total}

Além das proteínas, os fármacos também podem interagir com outros biopolímeros, como hemácias, as quais representam $45 \%$ do volume sanguíneo total. ${ }^{49}$ Alguns fármacos apresentam alta concentração eritrocítica (ex.: imunossupressores) tornando a análise do sangue total necessária para estudos de disposição cinética destes analitos. ${ }^{48}$ Da mesma forma que o plasma, a coleta do material requer um procedimento invasivo (punção venosa).

Normalmente, a análise de sangue total não requer nenhum tratamento prévio para extração (por exemplo, precipitação protéica) utilizando-se LPME. ${ }^{32}$ No entanto, devido à maior viscosidade do sangue total em relação ao plasma, o tempo necessário para atingir o equilíbrio de extração é ligeiramente maior. ${ }^{39}$

Halvorsen et $a l .{ }^{32}$ relataram a primeira aplicação da LPME em sangue total tendo metanfetamina e citalopram como fármacos-modelo, obtendo alta seletividade (livre de interferentes) e grau de enriquecimento comparável à análise de plasma. O mesmo grupo desenvolveu um método empregando a técnica aliada à espectrometria de massas para o "screening" de compostos anfetamínicos no sangue total. ${ }^{57}$ Mais tarde, nove antidepressivos foram determinados nesta matriz utilizando-se menores quantidades de amostra (50-500 $\mu \mathrm{L})$ e acetato de dodecila e ácido fórmico como componentes do sistema de três fases. ${ }^{58}$ Ugland et al. ${ }^{39}$ ofereceram uma visão geral das características da LPME na análise de sangue total, particularmente na otimização das condições de extração no modo de três fases. Neste trabalho, vários parâmetros foram estudados para determinação de fármacos de caráter básico neste fluido biológico em especial.

\section{$\underline{\text { Urina }}$}

A urina é uma das matrizes mais comumente estudadas na análise de fármacos, devido à relativa facilidade de coleta e, também, por ser a principal via de excreção de fármacos e metabólitos. É uma matriz com moderada complexidade e tipicamente contém compostos inorgânicos e orgânicos, com quantidade relativamente grande de sais. ${ }^{59,60} \mathrm{~A}$ eficiência na extração pode variar devido a diversas razões. Variações no pH da urina afetam a eliminação de muitos fármacos, assim como variações na força iônica alteram a viscosidade da amostra, promovendo efeitos significantes na extração. Geralmente, os fármacos são excretados na urina em grande quantidade, tornando a análise desta matriz bastante útil com a compensação dos menores valores de recuperação obtidos nas técnicas de microextração. Diversos trabalhos vêm sendo realizados utilizando urina como matriz (Tabela 1). Recentemente, De Oliveira et al. ${ }^{61}$ desenvolveram um método para análise enantiosseletiva da hidroxicloroquina e metabólitos em urina empregando $n$-octanol como solvente de extração e posterior análise por CE. Neste trabalho, valores de recuperação em torno de $80 \%$ foram observados e o método foi aplicado em um estudo piloto de disposição cinética, mostrando potencial para aplicação em estudos farmacocinéticos de excreção destes analitos. Um emprego importante utilizando urina como matriz é na área da toxicologia forense. De Jager et al. ${ }^{62}$ relataram a análise da cocaína e metabólitos em urina, utilizando clorofórmio como solvente de extração e análise por cromatografia gasosa. Constitui um método interessante pois o tempo de extração utilizado é de apenas 3 min, característica muito desejável em análises para fins médicos-legais, ou seja, alta rapidez.

\section{Leite materno}

O leite humano é uma matriz muito interessante pois serve como um meio de quantificar compostos que foram ingeridos previamente pela mãe e que podem, potencialmente, causar dano ao recém-nascido. É constituído por água (88\%), proteínas (3\%), lipídeos (aproximadamente $3 \%$ ) e carboidratos na forma de lactose $(6,8 \%) \cdot{ }^{63} \mathrm{O}$ colostro que aparece no início da lactação difere significativamente do leite habitual, pois contém menos lactose e praticamente não contém lipídeos. ${ }^{64}$ A composição do leite humano é constante a partir da terceira semana após o nascimento do bebê, contudo a composição do colostro pode variar muito a partir do início da lactação até a terceira semana. Essa variação pode levar a diferenças na eficiência da extração e, portanto, deve ser levada em consideração. Apenas uma aplicação é relatada empregando LPME na análise de leite materno. Bjorhovde et al. ${ }^{65}$ relataram a extração de quatro antidepressivos (paroxetina, fluvoxamina, mianserin e citalopram) nesta matriz. Esse trabalho é interessante pois demonstra o uso de um solvente jamais visto em LPME (silicone polifenil-metilsiloxano). O solvente utilizado promoveu uma grande estabilidade química ao sistema, porém os valores de recuperação inicialmente observados foram baixos (18-38\%). Acredita-se que os valores obtidos sejam devidos à complexidade da matriz e ao conteúdo de lipídeos presentes. Assim, após nova etapa de preparo de amostra, como centrifugação e adição de ácido clorídrico para supressão das interações entre os fármacos e a matriz, os valores de recuperação aumentaram para 42-69\%.

\section{$\underline{\text { Saliva }}$}

A saliva é uma matriz pouco explorada na análise de fármacos. Apesar de ser um material relativamente viscoso, o conteúdo de lipídeos e proteínas é pequeno quando comparado a outros fluidos. ${ }^{56}$ É uma matriz não-invasiva e de relativa facilidade de coleta. De Jager et al. ${ }^{66}$ relataram a extração de cocaína e metabólitos utilizando clorofórmio como solvente orgânico e posterior análise por GC. Neste trabalho, os tempos de extração (10 min) e de análise (5 min) tornam o método muito atraente para uso em centros de toxicologia. Uma consideração importante na análise de fármacos na saliva é a possível contaminação da cavidade bucal com outras substâncias. Assim, o trabalho citado avaliou a extração da cocaína e metabólitos na presença de contaminantes comuns, como creme dental, suco de laranja, anti-séptico bucal, pastilhas, bicarbonato de sódio e açúcar. Essas substâncias não apresentaram o mesmo tempo de retenção dos fármacos analisados, impossibilitando a ocorrência de falsos positivos. Por outro lado, os resultados mostraram que os possíveis interferentes diminuíram a quantidade extraída, porém não foram suficientes para impedir a extração e detecção dos analitos.

\section{$\underline{\text { Cabelo }}$}

O cabelo é uma matriz muito interessante para análise de fármacos, pois a coleta é relativamente não-invasiva e o conteúdo pode informar uma história de exposição por um longo período. Outra vantagem em utilizar o cabelo é que devido às características apolares da matriz, ocorre o acúmulo preferencial do fármaco na forma original em relação aos metabólitos (mais polares). Essas características são ideais para análise de fármacos que são extensivamente metabolizados e freqüentemente não detectados em outros tecidos. ${ }^{56} \mathrm{O}$ cabelo pode ser dividido em três camadas distintas. ${ }^{67}$ A camada mais externa (cutícula) é formada por escamas sobrepostas (células achatadas) com as extremidades livres direcionadas para a ponta do cabelo. O córtex encontra-se em posição intermediária, constituindo a maior parte do cabelo. Consiste de queratina dura empacotada firmemente, contendo pigmento e pequenos espaços de ar. A medula forma a parte mais interna consistindo de queratina e pode ser quebrada em pedaços descontínuos. Contém maiores espaços de ar, glóbulos de gordura e pigmentos. Yazdi e Es 'haghi ${ }^{26}$ desenvolveram um método para análise da morfina, codeína e metadona em cabelo. Os autores utilizaram $n$-octanol como solvente orgânico e posterior análise por HPLC. O trabalho é interessante pois 
mostra a aplicação de surfactantes não-iônicos utilizados para melhorar a eficiência da extração, com valores de recuperação finais do método entre $62-93 \%$.

\section{CONCLUSÕES}

Além das discussões demostrando os fundamentos da LPME, o trabalho também relacionou as mais importantes aplicações da técnica em matrizes biológicas descritas na literatura corrente. A LPME desponta como sendo extremamente atrativa, pois as unidades de extração apresentam baixo custo e, desta forma, possibilitam o uso único do dispositivo, evitando-se problemas de contaminação cruzada. Por sua vez, o consumo de solvente orgânico é praticamente eliminado e a técnica é compatível com uma grande variedade de amostras biológicas (sangue total, plasma, urina, saliva e leite materno) ou não (análise de água, solo e alimentos). A técnica pode ainda proporcionar de médias a elevadas recuperações, com elevado enriquecimento dos analitos, seletividade e excelente "clean-up" (limpeza) de amostras complexas. Além disso, as extrações são realizadas entre 15-45 min na maioria das aplicações. Devido à simplicidade e ao baixo custo das unidades de extração, muitas amostras podem ser processadas paralelamente, proporcionando um ganho no tempo total de análise.

Por fim, a LPME pode ser empregada no modo três fases, duas fases ou mediada por carreador utilizando exatamente as mesmas unidades de extração, o que proporciona um elevado grau de flexibilidade à essa técnica.

Trabalhos futuros, principalmente de caráter aplicado, poderão conferir maior credibilidade à técnica, confirmando-a como uma alternativa viável para aplicações diversas e que os resultados obtidos são comparáveis ou mesmo superiores àqueles obtidos empregando técnicas tradicionais de extração, como a LLE e SPE.

\section{REFERENCIAS}

1. Gilar, M.; Bouvier, E. S. P.; Compton, B. J.; J. Chromatogr., A 2001, 909, 111.

2. Queiroz, S. C. N.; Collins, C. H.; Jardim, I. C. S. F.; Quim. Nova 2001, $24,68$.

3. Kataoka, H.; Trends Anal. Chem. 2003, 22, 232.

4. Smith, R. M.; J. Chromatogr., A 2003, 1000, 3.

5. Saito, Y.; Jinno, K.; J. Chromatogr., A 2003, 1000, 53.

6. Arthur, C. L.; Pawliszyn, J.; Anal. Chem. 1990, 62, 2145.

7. Valente, A. L. P.; Augusto, F.; Quim. Nova 2000, 23, 523.

8. Theodoridis, G.; Koster, E. H. M.; Jong, G. J.; J. Chromatogr., B: Anal. Technol. Biomed. Life Sci. 2000, 745, 49.

9. Ulrich, S.; Kruggel, S.; Weigmann, H.; Hiemke, C.; J. Chromatogr., B: Anal. Technol. Biomed. Life Sci. 1999, 731, 231.

10. http://www.sigmaaldrich.com/Brands/Supelco_Home/Spotlights/SPME_ central.html, acessada em Outubro 2006.

11. Chong, S. L.; Wang, D.; Hayes, J. D.; Wilhite, B. W.; Malik, A.; Anal. Chem. 1997, 69, 3889.

11. Psillakis, E.; Kalogerakis, N.; Trends Anal. Chem. 2002, 21, 53.

12. Jeannot, M. A.; Cantwell, F. F.; Anal. Chem. 1996, 68, 2236.

13. Jeannot, M. A.; Cantwell, F. F.; Anal. Chem. 1997, 69, 235.

14. Pedersen-Bjergaard, S.; Rasmussen, K. E.; J. Chromatogr., B: Anal. Technol. Biomed. Life Sci. 2005, 817, 3.

15. Jönsson, J. Å.; Mathiasson, L.; Trends Anal. Chem. 1999, 18, 318.

16. Jönsson, J. Å.; Mathiasson, L. M.; LC-GC Europe 2003, 16, 683.

17. Jönsson, J. A.; Mathiasson, L.; J. Chromatogr., A 2000, 902, 205.

18. Jönsson, J. Å.; Mathiasson, L.; Trends Anal. Chem. 1999, 18, 325.

19. van de Merbel, N. C.; J. Chromatogr., A 1999, 856, 55.

20. Pedersen-Bjergaard, S.; Rasmussen, K. E.; Anal. Chem. 1999, 71, 2650.

21. Psillakis, E.; Kalogerakis, N.; Trends Anal. Chem. 2003, 22, 565.

22. Rasmussen, K. E.; Pedersen-Bjergaard, S.; Trends Anal. Chem. 2004, 23, 1.

23. Ho, T. S.; Halvorsen, T. G.; Pedersen-Bjergaard, S.; Rasmussen, K. E.; J. Chromatogr., A 2003, 998, 61 .

24. Ho, T. S.; Reubsaet, J. L. E.; Anthonsen, H. S.; Pedersen-Bjergaard, S.; Rasmussen, K. E.; J. Chromatogr., A 2005, 1072, 29.
25. Ho, T. S.; Pedersen-Bjergaard, S.; Rasmussen, K. E.; J. Chromatogr. Sci. 2006, 44, 308

26. Yazdi, A. S.; Es'haghi, Z.; J. Chromatogr., A 2005, 1094, 1.

27. Ho, T. S.; Pedersen-Bjergaard, S.; Rasmussen, K. E.; J. Chromatogr., A 2002, $963,3$.

28. http://www.membrana.com/index_neu.phtml, acessada em Outubro 2006.

29. Andersen, S.; Halvorsen, T. G.; Pedersen-Bjergaard, S.; Rasmussen, K. E.; J. Chromatogr., A 2002, 963, 303.

30. Pedersen-Bjergaard, S.; Rasmussen, K. E.; J. Sep. Sci. 2004, 27, 1511.

31. Basheer, C.; Balasubramanian, R.; Lee, H. K.; J. Chromatogr., A 2003, 1016, 11.

32. Halvorsen, T. G.; Pedersen-Bjergaard, S.; Rasmussen, K. E.; J. Chromatogr. B: Anal. Technol. Biomed. Life Sci. 2001, 760, 219.

33. Basheer, C.; Lee, H. K.; Obbard, J. P.; J. Chromatogr., A 2002, 968, 191.

34. Lambropoulou, D. A.; Albanis, T. A.; J. Chromatogr., A 2005, 1072, 55.

35. Shen, G.; Lee, H. K.; Anal. Chem. 2002, 74, 648.

36. Pedersen-Bjergaard, S.; Rasmussen, K. E.; Eletrophoresis 2000, 21, 579.

37. Pedersen-Bjergaard, S.; Ho, T. S.; Rasmussen, K. E.; J. Sep. Sci. 2002, 25 , 141.

38. Ulrich, S.; J. Chromatogr., A 2000, 902, 167

39. Ugland, H. G.; Krogh, M.; Reubsaet, J. L. E.; J. Chromatogr., B: Anal. Technol. Biomed. Life Sci. 2003, 798, 127.

40. Peng, G. W.; Chiou, W. L.; J. Chromatogr. 1990, 531, 3.

41. Kramer, K. E.; Andrews, A. R. J.; J. Chromatogr., B: Anal. Technol. Biomed. Life Sci. 2001, 760, 27.

42. Reubsaet, J. L. E.; Loftheim, H.; Gjelstad, A.; J. Sep. Sci. 2005, 28, 1204.

43. Esrafili, A.; Yamini, Y.; Shariati, S.; Anal. Chim. Acta 2007, 604, 127.

44. Kuuranne, T.; Kotiaho, T.; Pedersen-Bjergaard, S.; Rasmussen, K. E.; Leinonen, A.; Westwood, S.; Kostiainen, R.; J. Mass Spectrom. 2003, 38, 16.

45. Dietz, C.; Sanz, J.; Cámara, C.; J. Chromatogr., A 2006, 1103, 183.

46. Wu, J.; Ee, K. H.; Lee, H. K.; J. Chromatogr., A 2005, 1082, 121.

47. Pawliszyn, J.; Pedersen-Bjergaard, S.; J. Chromatogr. Sci. 2006, 44, 291.

48. Moffat, A. C.; Osselton, M. D.; Widdop, D.; Galichet, L. Y.; Clarke's Analysis of Drugs and Poisons, $3^{\text {rd }}$ ed., Pharmaceutical Press: London, 2004.

49. Shargel, L.; Yu, A. B. C.; Applied Biopharmaceutics and Pharmacokinetics, $3^{\text {rd }}$ ed., Prentice-Hall: New York, 1993.

50. Ugland. H. G.; Krogh, M.; Rasmussen, K. E.; J. Chromatogr., B: Anal. Technol. Biomed. Life Sci. 2000, 749, 85.

51. Ho, T. S.; Pedersen-Bjergaard, S.; Rasmussen, K. E.; Analyst 2002, 127, 608

52. Basheer, C.; Lee, H. K.; Obbard, J. P.; J. Chromatogr., A 2004, 1022, 161.

53. Andersen, S.; Halvorsen, T. G.; Pedersen-Bjergaard, S.; Rasmussen, K. E.; Tanum, L.; Refsum, H.; J. Pharm. Biomed. Anal. 2003, 33, 263.

54. De Santana, F. J. M.; De Oliveira, A. R. M.; Bonato P. S.; Anal. Chim. Acta 2005, 549, 96

55. Magalhães, I. R. S.; Bonato, P. S.; J. Pharm. Biomed. Anal., no prelo.

56. Lord, H.; Pawliszyn, J.; J. Chromatogr., A 2000, 902, 17.

57. Halvorsen T. G.; Pedersen-Bjergaard, S.; Reubsaet, J. L. E.; Rasmussen, K. E.; J. Sep. Sci. 2001, 24, 615.

58. Halvorsen T. G.; Pedersen-Bjergaard, S.; Reubsaet, J. L. E.; Rasmussen, K. E.; J. Sep. Sci. 2003, 26, 1520 .

59. Yashiki, M.; Nagasawa, N.; Kojima, T.; Miyazaki, Y.; Iwasaki, Y.; Jpn. J. Forensic Toxicol. 1995, 13, 17.

60. Dehon, B.; Humbert, L.; Devisme, L.; Stievenart, M.; Mathieu, D.; Houdret, N.; Lhermitte, M.; J. Anal. Toxicol. 2000, 24, 22.

61. De Oliveira, A. R. M.; Cardoso, C. D.; Bonato, P. S.; Electrophoresis 2007, 28, 1081.

62. De Jager, L. S.; Andrews, A. R. J.; Analyst 2001, 126, 1298.

63. Strand, F. L.; Physiology: A Regulatory Systems Aproach, Macmillan Publishing: New York, 1978.

64. Tortora, G. J.; Introduction to the Human Body, Harper and Row Publishers: New York, 1988.

65. Bjorhovde, A.; Halvorsen, T. G.; Rasmussen, K. E.; Pedersen-Bjergaard, S.; Anal. Chim. Acta 2003, 491, 155.

66. De Jager, L. S.; Andrews, A. R. J.; Anal. Chim. Acta 2002, 458, 311.

67. Romanes, G. J.; Cunningham's Textbook of Anatomy, $12^{\text {th }}$ ed, Oxford University Press: New York, 1981

68. Hou, L.; Wen, X.; Tu, C.; Lee, H. K.; J. Chromatogr., A 2002, 979, 163.

69. Halvorsen, T. G.; Pedersen-Bjergaard, S.; Rasmussen, K. E.; J. Chromatogr., A 2001, 909, 87.

70. Rasmussen, K. E.; Pedersen-Bjergaard, S.; Krogh, M.; Ugland, H. G.; Gronhaug, T.; J. Chromatogr., A 2000, 873, 3.

71. Leinonen, A.; Vuorensola, K.; Lepola, L. M.; Kuurane, T.; Kotiaho, T.; Ketola, R. A.; Kostiainen, R.; Anal Chim. Acta 2006, 559, 166.

72. Yamini, Y.; Reimann, C. T.; Vatanara, A.; Jönsson, J. A.; J. Chromatogr., A 2006, 1124, 57. 